\title{
Magnetic resonance elastography-new wave of hepatic imaging
}

\author{
Marilyn J. Siegel
}

(C) Springer-Verlag 2011

Magnetic resonance elastography (MRE) is a promising new imaging technique that noninvasively quantifies liver stiffness by using mechanical waves. The application of this technique for assessing liver fibrosis is based on the premise that stiffness of hepatic parenchyma increases as fibrosis increases.

\section{MRE technique}

MRE uses low-frequency mechanical waves to interrogate elasticity of tissue. There are three steps in performing MRE. First, a mechanical drumlike device is placed on the abdominal wall to generate shear waves (frequency 40 $120 \mathrm{~Hz}$ ). Next, the waves are imaged using a phase-contrast MR imaging sequence. Third, wave data are processed to create a color elastogram, an image that quantitatively depicts tissue stiffness. Liver stiffness is calculated by placing regions of interest in selected locations on the elastogram. The average acquisition time for hepatic MRE is approximately $15 \mathrm{~s}$, so the addition of MRE into a conventional MRI examination of the liver adds very little to the total examination time.

\section{Diagnosing hepatic fibrosis}

Normal liver tissue has a stiffness of approximately 2 kilopascals $(\mathrm{kPa})$, which indicates soft tissue and is similar

Disclaimer Dr. Siegel has no financial interests, investigational or off-label uses to disclose.

M. J. Siegel $(\bowtie)$

Mallinckrodt Institute of Radiology,

St. Louis, MO 63110, USA

e-mail: SiegelM@mir.wustl.edu to that of subcutaneous fat. Mean liver stiffness values increase with greater degrees of liver fibrosis. In one study of adults, the mean liver stiffness was $2.2 \mathrm{kPa}$ for a healthy control group and $5.8 \mathrm{kPa}$ for biopsy-proven liver disease group. When a $2.93 \mathrm{kPa}$ value was used as the upper limit of normal for liver tissue stiffness, MRE had a sensitivity of $98 \%$ and specificity of $99 \%$ for differentiating fibrosis from normal liver tissue, with a negative predictive value of $97 \%$. When liver stiffness was more than $4.89 \mathrm{kPa}$, sensitivity and specificity were $86 \%$ and $85 \%$, respectively, for identifying patients with moderate to severe fibrosis compared with early fibrosis. Hepatic stiffness was not influenced by the presence of hepatic steatosis.

In our pediatric experience, the mean hepatic shear stiffness in children with cirrhosis was significantly greater than in those without cirrhosis $(P<0.005)$ (Figs. 1 and 2). Receiver-operator characteristic analysis identified a cutoff of $3.57 \mathrm{kPa}$ as being $100 \%$ sensitive and specific both for cirrhosis in this population. Intrahepatic triglyceride was increased in six patients (mean 9.6\%) and showed no relationship to cirrhosis (Siegel personal experience).

\section{Limitations of MRE}

MRE analysis of liver fibrosis could conceivably be confounded by factors that can alter liver stiffness, such as hepatic inflammation, vascular congestion, cholestasis and portal hypertension. More studies will be needed to determine the extent to which these factors affect the accuracy of MRE for diagnosing fibrosis. Another technical limitation is moderate to severe hepatic iron overload. The signal intensity of the liver may be so low that the shear waves cannot be seen on the phase-contrast gradient-echo images. 


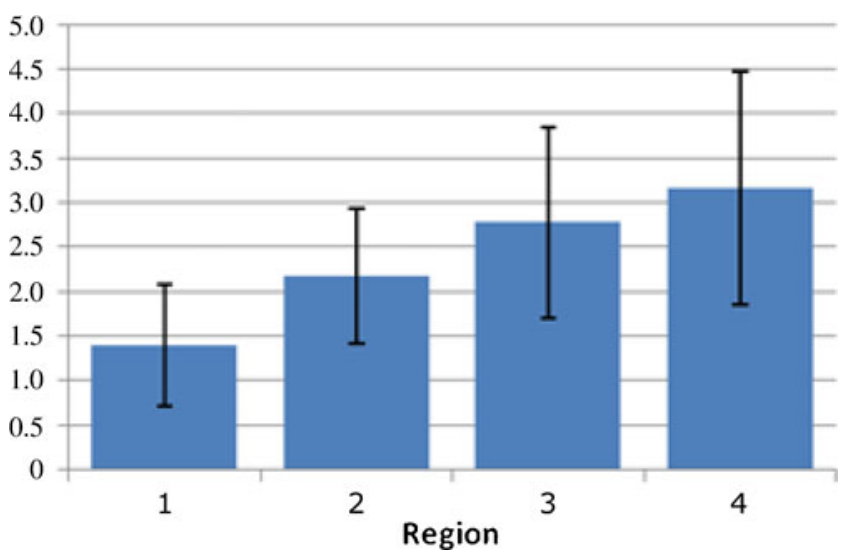

Fig. 1 Normal liver. All $\mathrm{kPa}$ values are below 3

\section{Clinical role of MRE in chronic hepatic disease}

MRE appears to have high diagnostic accuracy for assessing hepatic fibrosis and thus may have a future role in selecting patients for biopsy to assess potential hepatic fibrosis. Another clinical application may be the longitudinal monitoring of patients being treated for fibrosis.

\section{US elastography in hepatic fibrosis}

US transient elastography using pulse-echo US signals is an alternative method of assessing hepatic fibrosis. Similar to MRE, results are expressed in units of kilopascals. This technology has sensitivity of approximately $85 \%$ and specificity of $>90 \%$ for detecting cirrhosis. US elastography requires an acoustic window and can be limited in the

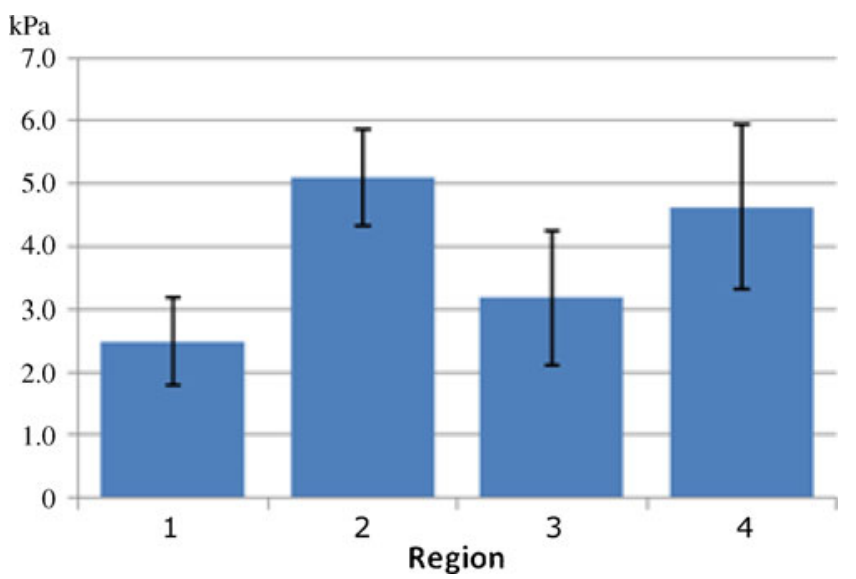

Fig. 2 Hepatic fibrosis. Some values range above normal $(>3 \mathrm{kPa})$

presence of obesity or ascites. In addition, it samples only a small volume of the liver. By comparison, MRE is not affected by obesity or ascites and it provides a global view of the liver and is thus potentially less affected by sampling error.

\section{MRE in diagnosing focal hepatic lesions}

MRE has been used to characterize liver tumors, including metastatic lesions, hepatocellular carcinomas, hemangiomas, focal nodular hyperplasia and hepatic adenoma. Malignant liver tumors have significantly higher mean shear stiffness values $(10.1 \mathrm{kPa})$ than benign tumors $(2.7 \mathrm{kPa})$ and normal liver tissue. Preliminary data suggest that MRE is a useful sequence to complement traditional MRI criteria in distinguishing between benign and malignant lesions. 